\title{
COLD STORAGE-SUPPORTED AIR CONDITIONING SYSTEM IN URBAN TRANSPORT VEHICLES
}

\author{
Wojciech Jarzyna ${ }^{1}$, Dariusz Zielinski ${ }^{1}$, Michał Aftyka $^{1}$, Karol Fatyga $^{1}$ \\ 1 Electrical Engineering and Computer Science, Lublin University of Technology, Lublin, Poland, e-mail: \\ w.jarzyna@pollub.pl
}

Received: 2016.08.16

Accepted: 2016.09.26

Published: 2016.11.01

\begin{abstract}
A bottleneck for the development of public transport vehicles is their electricity supply. Electric buses are almost exclusively equipped with electrochemical batteries, while nearly $40 \%$ of the energy is used in the processes of air conditioning. For this reason, we developed and built a demonstration system for storing thermal energy in public transport vehicles. The most important effects are: significant reduction of financial expenses and of the total weight of all batteries with the same amount of stored energy.
\end{abstract}

Keywords: thermal energy storage, electric city bus, energy management

\section{INTRODUCTION}

Electric vehicles have longer history than petrol driven ones, and at the same time a number of indisputable advantages over their competitors. Despite this, for more than a hundred years it has been difficult for them to move to the top in road transport. The limitation is the energy available on board of the vehicle. For many decades, this problem was practically insoluble. Most lead-acid batteries were too heavy and had too little energy capacity to provide a transfer over a satisfactory distance. Currently, this problem is increasingly overcome. There are different types of rechargeable power source, for example: lithium-ion, lithium-ion polymer, nickel cadmium, alkaline, nickel-metal hydride (Cuma \& Koroglu, 2015) and super capacitors. Their use has significantly increased the range of electric vehicles (Thounthonget al., 2009). Despite this, the amount of energy that can be stored on board of an electric vehicle is lower than in combustion engine vehicles.

The problem is visible even more clearly in bus transport. Frequent starts, braking and exchange of passengers at bus stops all increase energy demand. It cannot be solved by simply increasing the capacity of rechargeable electrochemical batteries, because the additional weight reduces the number of passengers to be transported. For this reason, the problem was addressed in an unconventional way. It was taken into account that in the process of air conditioning the output energy is thermal energy. Therefore, the possibility of accumulating heat in the form of cooled or heated medium was considered (Oró et al., 2012).

These questions are addressed in this paper. It presents a description of the construction and operation of the air-conditioning system with storage tanks of cool/heat, the idea which was reported in a patent claim (Zielinski et al., 2016). It includes the discussion of the materials and components used in the construction of an experimental station located in the Laboratory for the Conversion and Utilisation of Energy from Renewable Sources at the Lublin University of Technology. Of particular importance are the results of tests carried out to determine the basic parameters of the system under study and the potential applications of the corresponding solutions in electric buses.

\section{THE IDEA OF THE EXPERIMENTAL DEMONSTRATOR}

The most common way to accumulate cold is the use of temperature changes in the heat-accu- 
mulating material called - sensible heat. Another way is to use the energy of phase transformation of the heat-accumulating material. Such a method of heat storage is described as latent heat (Fig. 1).

This phenomenon can be advantageously used for heat storage in heat-insulated accumulators. The energy absorbed or released during such a process is generally many times greater than the energy required to change the temperature of the substance. For example, freezing of $1 \mathrm{~m}^{3}$ of water at $0{ }^{\circ} \mathrm{C}$ should provide approximately 93 $\mathrm{kWh}$ of thermal energy (Khan et al., 2016). This amount of energy is accumulated as latent heat. However, in order to store the same energy in 1 $\mathrm{m}^{3}$ of water, its temperature should be changed almost by $80^{\circ} \mathrm{C}$ (Fig. 2).

Various substances have varying temperatures and freezing points of the phase change (Khan et al., 2016). By adding so-called eutectic mixtures, accumulating materials can be obtained with sim-

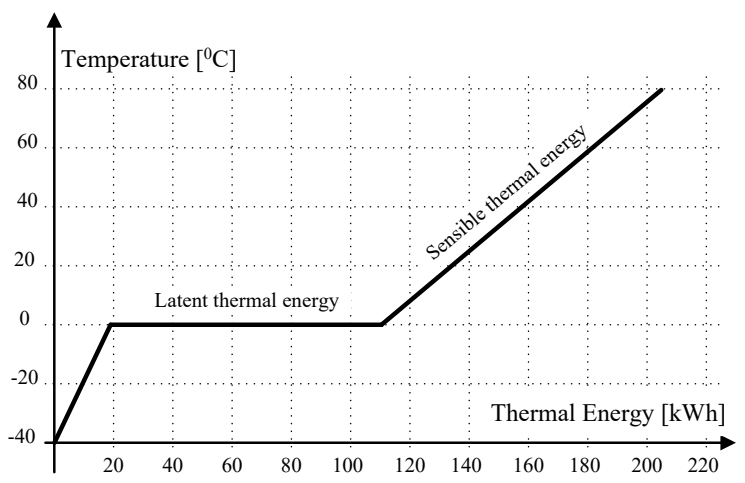

Fig. 1. Sensible and latent heat for $1 \mathrm{~m}^{3} \mathrm{H}_{2} 0$ during the phase transition ice-water

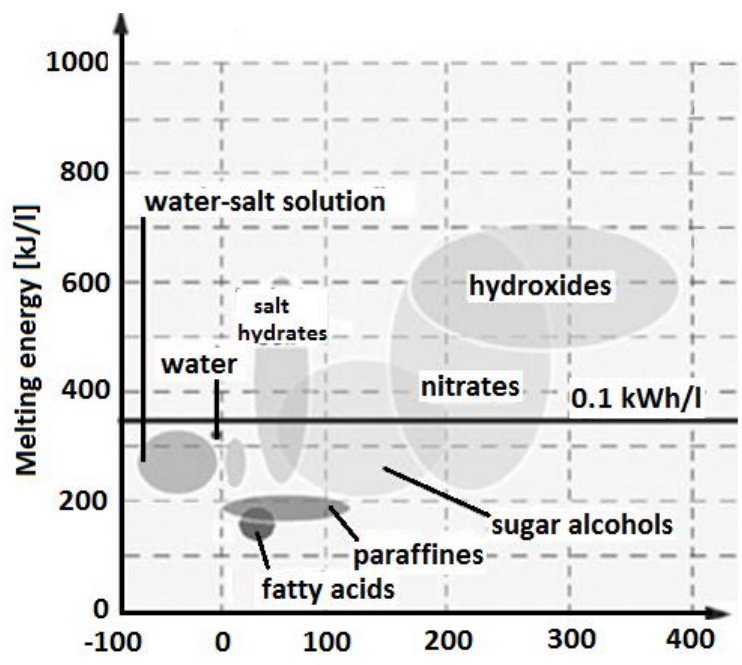

Fig. 2. Ability to store energy by selected material accumulation ilar thermal properties, differing only in change of state temperatures. Such materials can be salt hydrates in a mixture with water, e.g. $\mathrm{NaCl}, \mathrm{LiCl}$, $\mathrm{KCl}, \mathrm{MgCl}_{2}$, described as phase change materials (Oró et al., 2012).

Salt hydrates have a high value of latent melting heat per volume unit, a relatively high thermal conductivity and small changes in volume during melting. Therefore, these substances are also well suited for the storage of thermal energy (Sharma et al., 2007).

The common technical solutions typically use water as the substance storing thermal energy. However, the use of water in systems accumulating latent heat, presents technical problems connected with the increase of its volume during freezing. Such a phenomenon results in high stresses in the construction of heat accumulators. Therefore, the water-filled containers have a special structure which makes allowances for changes of the energy-accumulating medium. Interesting indications in this area can be found in (Donnelly et al. 2012), (Oró et al. 2012) or (Zielinski et al. 2016).

In the developed prototype storage design the substance storing thermal energy is water. In order to cool it, a significantly modified air conditioning system was used, which is a prototype research demonstrator design. The standard vehicle air conditioning system is composed of elements such as compressor, condenser, drying filter, evaporator and expansion valve. All these elements form a closed circuit of refrigerant and oil. In the basic cycle electrical energy is converted into thermal energy from the cooled medium. Thermodynamic transformations occur in a continuous manner, and the heat is not stored anywhere. In order to expand the possibilities of using such devices, a new system with a cold storage tank has been proposed (Zielinski et al., 2016). Its block diagram is composed of two circuits (Fig. 3):

- Hydraulic circuit, equipped with a circulating pump, control valves, cold accumulator, heat accumulator and exchanger. In the start-up system, this structure was filled with a water glycol solution with a very low freezing point of up to $-35^{\circ} \mathrm{C}$.

- Cooling circuit equipped with electronic expansion valves, condenser-evaporator, compressor, heat exchanger and valves changing the direction of refrigerant circulation. This circuit can operate in two modes, a refrigera- 


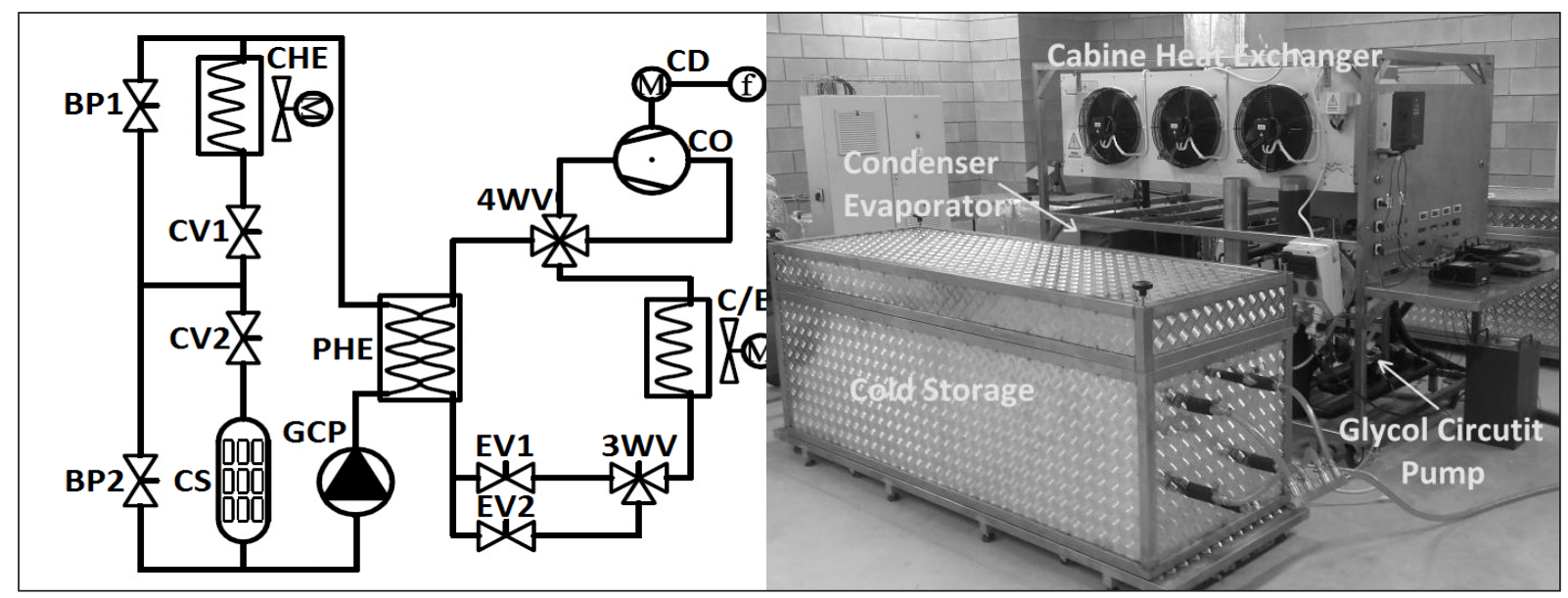

Fig. 3. Experimental demonstrator with storage of heat and cold, where: CHE - cabin heat exchanger, C/E - condenser/evaporator, CS - cold storage, $\mathrm{PHE}$ - plate heat exchanger, $\mathrm{CO}$ - compressor, $\mathrm{GCP}$ - glycol circuit pump, CD - compressor drive, EV1, EV2 - expansion valves, CV1, CV2 - controlled valves, BP1, BP2 - controlled BY-PASS valves, $4 \mathrm{WV}$ - four-way valve, $3 \mathrm{WV}$ - three-way valve

tion system or heat pump. Both circuits are coupled together by means of a plate heat exchanger, ensuring the separation of the media and heat exchange. This design allows the free flow of heat between the circuits.

An important role in the operation of the system is fulfilled by carefully selected control valves and a control algorithm. A detailed description of the construction can be found in the patent claim (Zielinski et al., 2016). For example, valves are used in the refrigeration circuit, changing the direction of the refrigerant circuit. This solution enabled the delivery of thermal energy from the heat accumulator to the interior of the bus. This energy can also be removed from the passenger compartment of the bus and transferred to the cold accumulator.

This device is powered by electricity, which can be drawn from the network at bus stops. However, the possibility of using the braking energy is of particular interest.

The system is designed in such a way so as to be able to receive a large amount of energy supplied e.g. during regenerative braking in a short time. As previously mentioned, the storage of heating or cooling energy is done in a system with a thermally insulated tank installed as part of the bus. The tank comprises cylindrical containers filled with water or an aqueous solution having a high capability of energy accumulation. These containers are made of a material providing mechanical strength and being a good heat flow moderator. They are immersed in waterglycol, whose task is to receive or deliver heat energy from the bus. The advantage of this is that the water containers have an increased effective heat exchange surface. The prototype thermal energy storage tank is shown in Figure 4.

Due to the dynamic operating requirements, a scroll type compressor was applied, allowing operation with widely variable angular velocity. In contrast to the piston compressors, its advantage is the possibility of operation with variable revolution speed of the spiral while keeping full lubrication. Such a solution can significantly reduce the energy consumption of the entire system in a city bus by maintaining the most favourable pressure parameters affecting the efficiency of the work (Aprea et al., 2006). Thanks to this the input power is reduced to the energy consumed by the compressor.

Important elements of the system are electronic control valves. They enable smooth and dynamic expansion pressure control in full range. Due to these properties, this type of valves allows to precisely regulate the temperature of the refrigerant circuit. Such operational comfort is not provided by cheap mechanical valves which open much more slowly and do not guarantee highprecision control. The quality of the work of the expansion valves applied is confirmed by their operating characteristics (Figure 5a).

The narrow hysteresis loop shown on the characteristics of Figure 5 promotes the uniqueness of the valve opening relative to the driving value. 


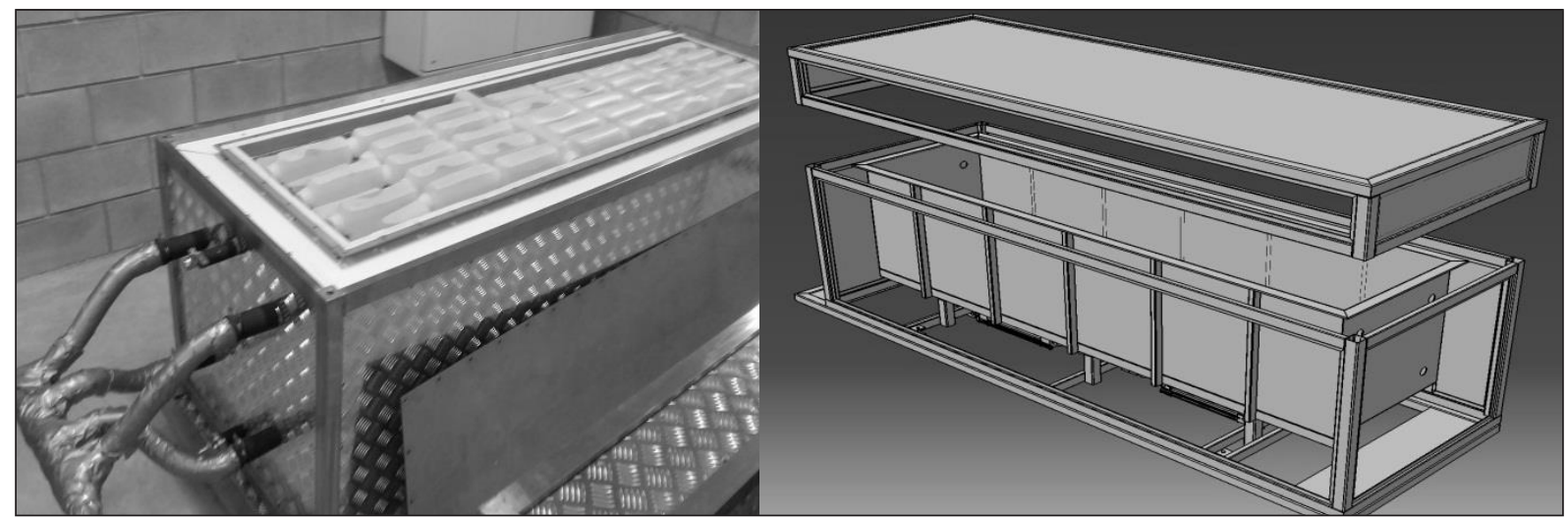

Fig. 4. a) Cold tank with lid ajar. Inside it are containers with water, b) 3D visualisation of the tank showing the requirements for thermal insulation.

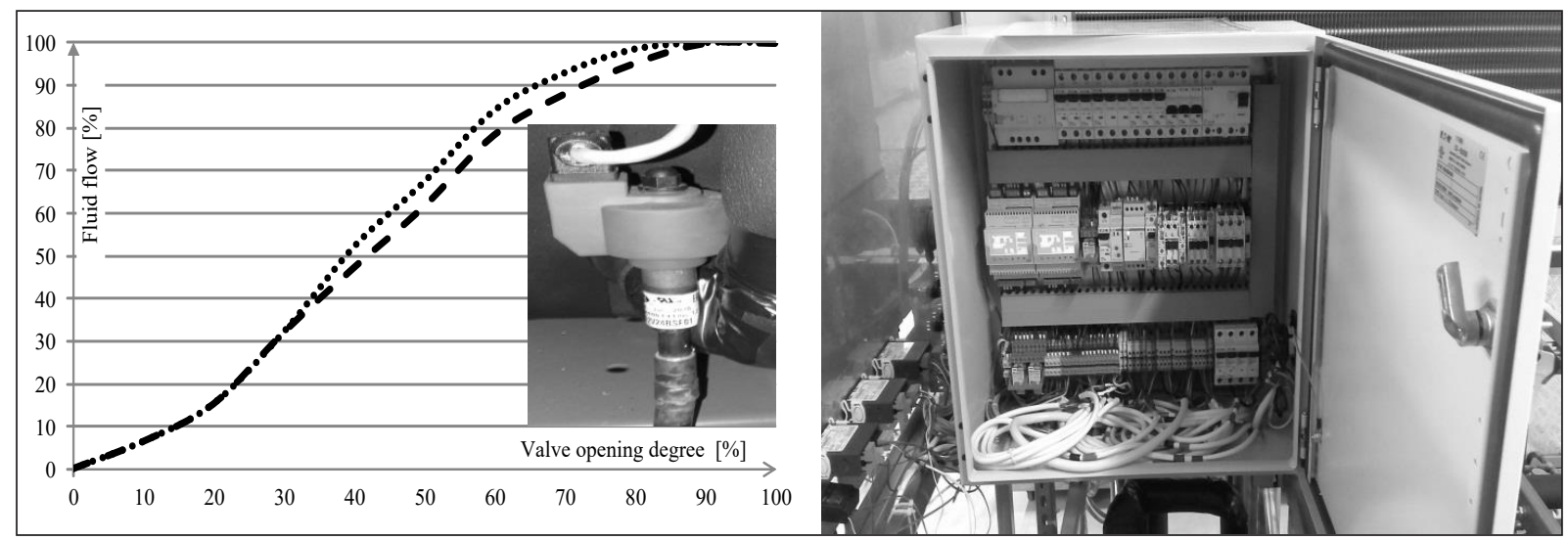

Fig. 5. a) Performance characteristics of electronic expansion valves during pressing and decompression,

b) Picture of the expansion valve and the flow control adjustment driver.

\section{TEST RESULTS}

In order to determine the accumulation possibility of the cold storage, and to explore the dynamics of the charging process, two cases were considered. In the first case, the applied frequency of the compressor drive system was $30 \mathrm{~Hz}$, which corresponds to the speed of the compressor motor of $885 \mathrm{rpm}$. Then the second case was examined, when the drive of the compressor was running at the rated capacity which corresponds to a frequency of $50 \mathrm{~Hz}$ and the engine speed of $1,475 \mathrm{rpm}$. Moreover, studies have to clarify whether the accumulation of energy in the form of latent heat is competitive relative to electrochemical energy sources, and whether fast loading of cartridges is possible. For both considered cases, the constant condensing temperature was set at $33{ }^{\circ} \mathrm{C}$. The electronic control system of the expansion valve worked at the highest efficiency and maintained the temperature of the refrigerant vapours superheating at $10{ }^{\circ} \mathrm{C}$. During the 140 -minute test, $6.28 \mathrm{kWh}$ was accumulated for the reduced cooling capacity and $12.16 \mathrm{kWh}$ for the full capacity of the system. In order to determine the amount of energy accumulated, a heat meter was used measuring the flow and temperature difference between the input and output of the cold tank tested. The quantity of energy stored was determined by the equation (1):

$$
Q=\int\left[\dot{m} \cdot c_{w} \cdot\left(t_{z}-t_{p}\right)\right] d t
$$

where: $Q$ - amount of heat $[\mathrm{J}]$

$\dot{m}$ - mass flow of liquid medium $[\mathrm{kg} / \mathrm{s}]$

$c_{W}$ - specific heat of the heating medium [J/(kg deg) $]$

$t_{z}$ - supply temperature of the medium (momentary measurement) [deg]

$t_{p}$ - return temperature of the medium (momentary measurement) [deg]

In the system under study heat is not measured directly, but calculated by the counting (integrating) circuit as the sum of the products of the momentary measurements of the difference in 
supply and return temperature of the heating medium and its mass flow.

On the basis of measurements of the flow of glycol, lower increases of the accumulated energy in the final working periods of the chiller were noted. This is associated with a reduction in the flow of fluid in the hydraulic system (Fig. 6b). The reason for the reduction in the accumulation capacity is a significant increase in the kinematic viscosity of the aqueous solution of glycol, which changed 28 times to lower the temperature from $+6{ }^{\circ} \mathrm{C}$ to $-4{ }^{\circ} \mathrm{C}$.

By performing simultaneous analysis of energy efficiency ratio EER during cooling process (Fig. 7a) and the temperature of the media inside the plate heat exchanger, the icing in the final testing phase, surface active plate heat exchanger, which resulted in a rapid loss of the system's ability to exchange energy, can be detected. The lower operating range of the aqueous solution of glycol used in this case is $-35^{\circ} \mathrm{C}$, and the evaporation temperature of the medium in the final phase of the test was $-44.3{ }^{\circ} \mathrm{C}$.

The performance graph in Figure 7a, enables to distinguish three states of the system operation:
- Operation with peak performance, which in the case under examination was 2.43 EER (removing heat in sensible form from the storage tank),

- Operation with constant cooling capacity the system is stable; this is due to the constant temperature of reception, which takes place during the phase transition (removing heat in latent form from the storage),

- Operation in an unacceptable area - the efficiency factor is less than a unity (a state of deep freezing of the storage, or icing of the plate heat exchanger).

While analysing the characteristics of the temperature at the input and output of Figures $8 \mathrm{a}$ and $8 \mathrm{~b}$, no area was observed where the temperature was periodically constant, which could point to a full heat balance of the system. Lack of temperature stability causes a strong dependence of the flow of water glycol solution as a function of the temperature of the plate heat exchanger, and the cooling tank charge. The increase in the temperature of the hydraulic fluid, which took place in the final testing phase is noteworthy. This phe-
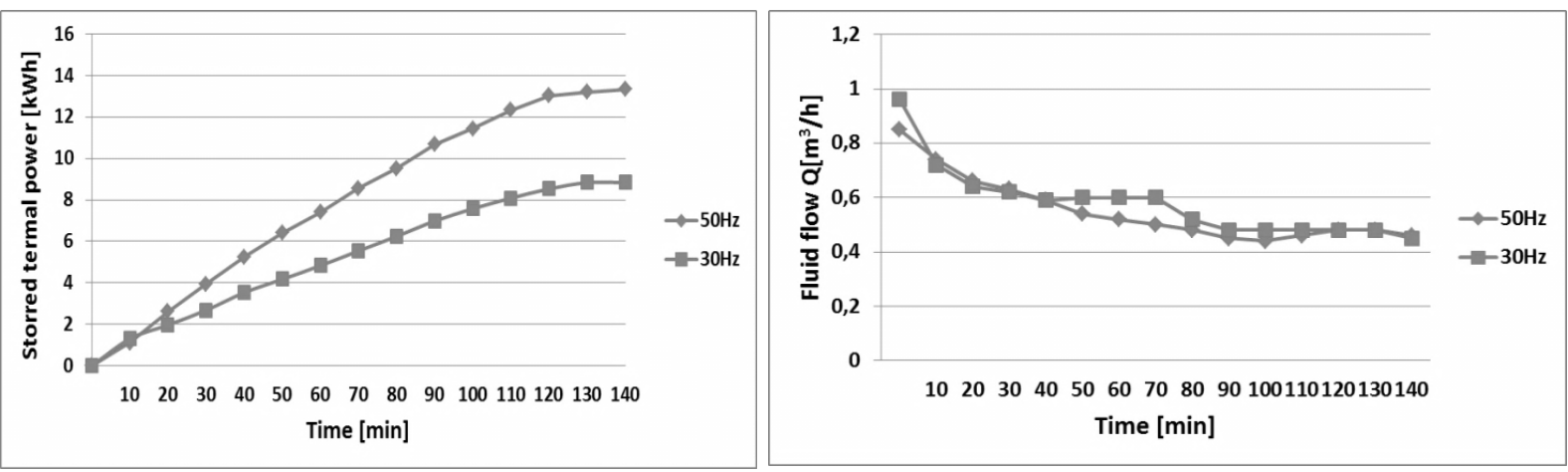

Fig. 6. a) Characteristics of the energy stored in the cooling tank as a function of time.

b) Characteristics of the flow of heat exchange medium as a function of time.
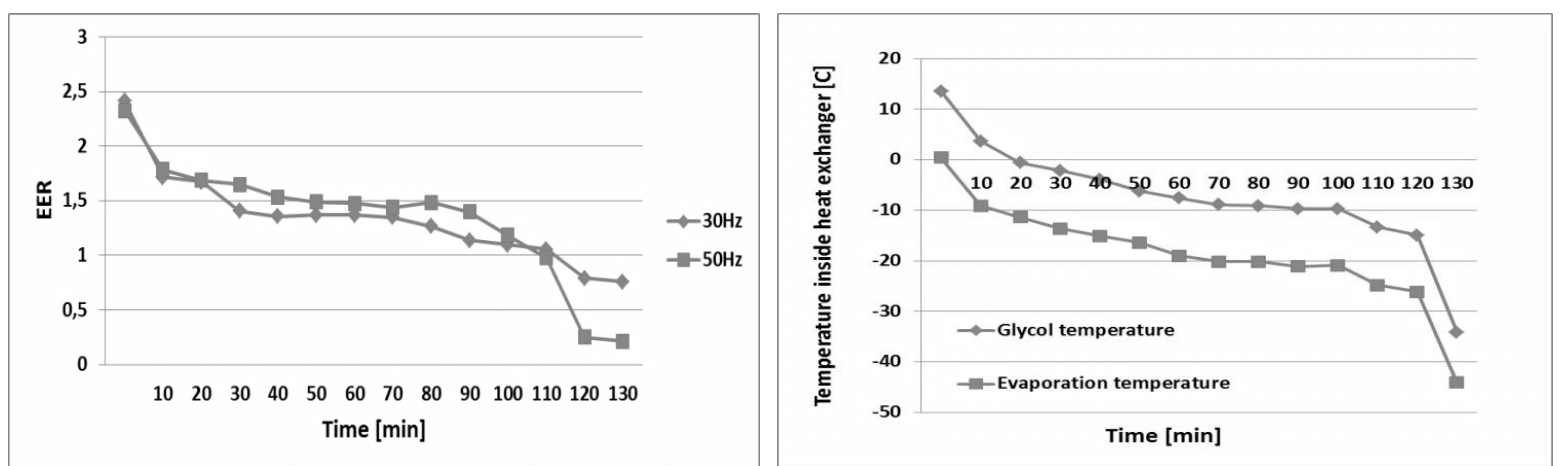

Fig. 7. Characteristics of the energy efficiency ratio during cooling of a tank as a function of time. b) Characteristics of the evaporation temperature of the medium and the temperature inside the heat exchanger plate. 


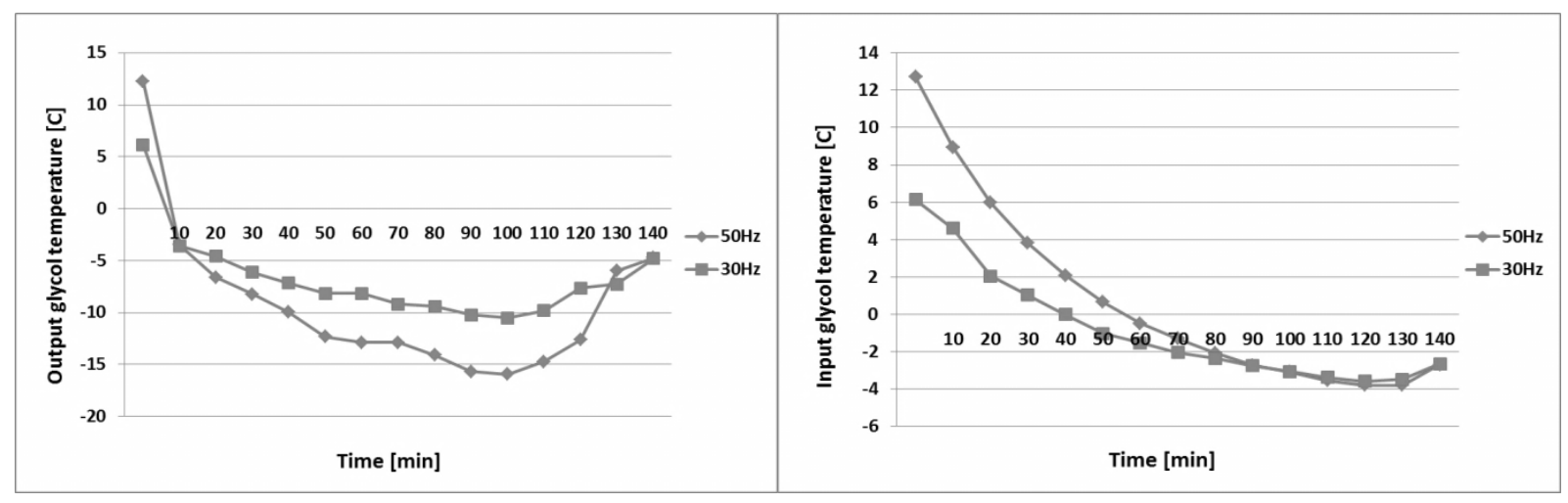

Fig. 8. Characteristics of coolant temperature as a function of time:

a) at the input of the heat exchanger, b) at the output of the exchanger

nomenon is caused as a result of two consecutive events. Analysing the final phase of the tank cooling cycle a declining ability of heat dissipation by the tank charges can be observed. This is quite natural, just as in the final stages of electrochemical battery charging. The difference between the source and the receiver temperature (potential) is reducing, which results in the lowering of the ability to receive energy. In the case of refrigeration systems, power can be continuously recovered from the hydraulic circuit, resulting in a rapid lowering of the temperature of the medium such as an aqueous solution of glycol and a reduction in the evaporation temperature of the refrigerant. The result is the icing of the heat exchange surface and introduction into the system of additional thermal resistance. In the event of such a situation, the system almost immediately lowers the efficiency ratio EER to a value less than a unity. In addition, the medium in the hydraulic circuit stops receiving the cold due to the aforementioned additional thermal resistance, which in turn results in an increase in the temperature of the hydraulic medium, which has ceased to be cooled down.

\section{QUICK ANALYSIS OF INVESTMENT AND OPERATING COSTS}

The rationale for the investment is as a rule determined by economic factors and safety considerations. This section compares the costs of buying an electrochemical battery with the costs of manufacturing the storage thermal tank collaborating with the distribution on-board thermal energy system. The calculation is that the energy demand of an electrical bus for thermal power be- tween consecutive recharges is about $13.34 \mathrm{kWh}$. The cold storage is therefore designed for this order of magnitude. According to the prices of 2016 in Poland, the cost of manufacturing a prototype system to store thermal energy with a capacity of $14 \mathrm{kWh}$ amounted to approximately $€ 1,600$. The stated amount covers only a cold storage tanks, and the cost does not include auxiliary equipment, such as the accumulator charging system. If serial production of such an arrangement of cold storage were to be started, the price of such a system would certainly be even lower.

Assuming that the average cooling efficiency of the air conditioning system for the urban cycle bus ride is EER $=1.5$, to produce in an electric

Table 1. The description of the materials and their parameters used in the construction of a thermal storage tank with a capacity of $14 \mathrm{kWh}$

\begin{tabular}{|l|c|}
\hline \multicolumn{1}{|c|}{ PCM material } & Distilled $\mathrm{H}_{2} \mathrm{O}$ \\
\hline $\begin{array}{l}\text { Own needs of cold storage } \\
\text { (circulation of cooling medium) }\end{array}$ & $35 \mathrm{Wh}$ \\
\hline Mass of the refrigerant input & $157 \mathrm{~kg}$ \\
\hline Total mass of the prototype & $312 \mathrm{~kg}$ \\
\hline Unit price & $120 € / \mathrm{kWh}$ \\
\hline
\end{tabular}

Table 2. Features of a lithium-ion battery with a capacity of $13.34 \mathrm{kWh}$

\begin{tabular}{|l|c|}
\hline \multicolumn{1}{|c|}{ Electric vehicle } & Solaris Trollino 18 \\
\hline Battery manufacturer & Wamtechnik \\
\hline Cell producer & EnerDel \\
\hline Rated capacity & $62 \mathrm{Ah}$ \\
\hline Nominal voltage & $613 \mathrm{~V}(3.65 \mathrm{~V} / \mathrm{VPC})$ \\
\hline Nominal energy & $38 \mathrm{kWh}$ \\
\hline Maximum charging current & $200 \mathrm{~A}$ \\
\hline Battery mass & $654 \mathrm{~kg}$ \\
\hline Unit price & $701.20 € / \mathrm{kWh}$ \\
\hline
\end{tabular}


city bus $13.34 \mathrm{kWh}$ of thermal energy, $8.9 \mathrm{kWh}$ of electricity is required. Such a low performance coefficient is related to the difficulty of cooling the condenser, which is located on the roof of the bus, whereby the air conditioning system is strongly dependent on the adverse effects of the weather, i.e. the roof exposure to strong sunshine or obstruction of air flow (Lee et al., 2012). The economic analysis was carried out on the basis of a Solaris vehicle, equipped with a package of cells with the following parameters:

While analysing the cost of electrochemical batteries, one should consider meeting the guarantee requirements, which in the case of an electric vehicle is at least 10 years. This forces the use of correction factors, which limit the operating range of cells to $50 \%$ of their nominal power. The calculation of the required operational capacity of lithium-ion cells included losses of energy conversion determined by the relation (2):

$$
Q_{B}=Q_{N} \cdot C_{B} \cdot \eta_{D C / D C} \cdot \eta_{D C / A C}
$$

where: $Q_{B}$ - operational capacity of the battery

$Q_{N}$ - rated capacity of the battery pack

$C_{B}-$ correction factor taking into account battery life

$\eta_{D C / D C}-$ efficiency of DC/DC boost converter

$\eta_{D C / A C}-$ efficiency of board inverter

Assuming that the nominal efficiency of the inverter of an air conditioner drive is 0.92 and the efficiency of the converter boosting the voltage of a lithium-ion battery reaches 0.94 , one can calculate that the required operational capacity of the battery pack should be $20.58 \mathrm{kWh}$. Such a pack of lithium-ion batteries has a mass of $354 \mathrm{~kg}$, while the cost of such a solution is estimated at $€ 14430$.

The above-mentioned analysis shows that the cost of a cold storage tank is only $11 \%$ of the cost of electrochemical batteries. The reservoir designed is therefore many times cheaper than electrochemical batteries, at approximately the same weight.

In addition, it is worth pointing out the time of operation. For comparable lithium-ion batteries it is 10 years, while maintaining the discharge up to $50 \%$. It follows that, in the long term, investment expenses on electrochemical accumulators increase significantly compared to thermal ones, because the life of the latter is estimated at about 30 years.

\section{CONCLUSIONS}

On the basis of the tests performed, it can be seen that the efficiency impact of the tested system for the storage of thermal energy depends largely on the capability to transfer energy in the hydraulic system. Hence, it is very important that the flow resistance be as low as possible. On the other hand, the glycol flow speed at the test stand has been significantly reduced through the installation of a large number of measuring devices, whereby the average EER does not exceed 1.5. In order to improve future operating parameters it is recommended that control of the flow speed of glycol be held in a feedback control system.

Very promising results have been achieved in terms of the parameters of the collection of thermal energy in the city bus. The solution in the form of a thermal storage tank is an interesting alternative to electrochemical cells and may contribute to reducing the cost of transport. Future research will be conducted on increasing the ERR efficiency coefficient of the prototype system and the replacement of the medium of an aqueous solution of glycol with a substance with a comparable specific heat and a low freezing point, which also maintains a low coefficient of kinetic viscosity.

\section{REFERENCES}

1. Cuma M.U. \& Koroglu T. 2015. A comprehensive review on estimation strategies used in hybrid and battery electric vehicles, Renewable and Sustainable Energy Reviews, Vol. 42, 517-531.

2. Oró E., Gracia A., Castell A., Farid M.M. \& Cabeza L.F. 2012. Review on phase change materials (PCMs) for cold thermal Energy storage applications: Applied Energy, 99, 513-533.

3. Zhiyuan W., Zhiliang X., Fengling G. \& Shengjun R. 2010. Experimental Study on Flow Characteristics of the Electronic Expansion Valve with Variable Condition, Asia-Pacific Power and Energy Engineering Conference, 1-4.

4. Donnelly E. 2012. Comparison of Ice-Bank Actual Results Against Simulated Predicted Results in Carroll Refurbishment Project DKIT: Journal of Sustainable Engineering Design, 1(2), Art. 3.

5. Sharma A., Tyagi Chen V.V. \& Buddhi D. 2007. Review on thermal energy storage with phase change materials and applications. Elseevier, 9 October 2007. 
6. Thounthong P., Raëlb S. \& Davatb B. 2009. Energy management of fuel cell/battery/supercapacitor hybrid power source for vehicle applications, Journal of Power Sources, 193(1), 376-38.5.

7. Khana Z., Khana Z. \& Ghafoor A. 2016. A review of performance enhancement of PCM based latent heat storage system within the context of materials, thermal stability and compatibility, Energy Conversion and Management, Vol. 115, 132-158.
8. Lee M.Y., Lee H.S. \& Won H.P. 2012. Characteristic Evaluation on the Cooling Performance of an Electrical Air Conditioning System Using R744 for a Fuel Cell Electric Vehicle. Energies, 5, 1371-1383. Doi:10.3390/en5051371.

9. Zielinski D., Jarzyna W. \& Kolano K. 2016. Method and system of storing heat or cold in vehicles with electric propulsion. Patent Assignee, Lublin University of Technology. 